\title{
Neurally adjusted ventilatory assist in neonates with congenital diaphragmatic hernia
}

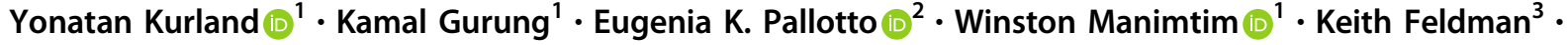 \\ Vincent S. Staggs $\mathbb{1}^{4} \cdot$ William Truog ${ }^{1}$
}

Received: 17 December 2020 / Revised: 27 April 2021 / Accepted: 6 May 2021 / Published online: 10 June 2021

(c) The Author(s), under exclusive licence to Springer Nature America, Inc. 2021

\begin{abstract}
Objective To measure short-term outcomes of neonates with congenital diaphragmatic hernia (CDH) while on Neurally Adjusted Ventilator Assist (NAVA), and to measure the impact of a congenitally abnormal diaphragm on NAVA ventilator indices.

Study design First, we conducted a retrospective-cohort analysis of 16 neonates with CDH placed on NAVA over a treatment period of $72 \mathrm{~h}$. Second, we performed a case-control study comparing NAVA level and Edi between neonates with $\mathrm{CDH}$ and those without $\mathrm{CDH}$.

Results Compared to pre-NAVA, there were clinically meaningful improvements in PIP $(p<0.003)$, Respiratory Severity Score $(p<0.001)$, MAP $(p<0.001)$, morphine $(p=0.004)$, and midazolam use $(p=0.037)$. Compared to a $1: 2$ matched group without $\mathrm{CDH}$, there was no meaningful difference in NAVA level $(p=0.286)$, Edi-Peak $(p=0.315)$, or Edi-Min $(p=0.266)$.

Conclusions The potential benefits of NAVA extend to neonates with CDH. There is minimal compensatory change in Edis, and higher/lower ventilator settings compared to neonates without $\mathrm{CDH}$.
\end{abstract}

\section{Introduction}

Neonates with congenital diaphragmatic hernia (CDH) present with a unique combination of non-formed or partially formed hemi-diaphragm, variable degree of concomitant pulmonary hypoplasia, pulmonary hypertension, and abnormal pulmonary innervation [1]. Consequently, many of these neonates require treatment with ventilation

Yonatan Kurland

ykurland@cmh.edu

1 Division of Neonatology, Children's Mercy Kansas City, Kansas City, MO, USA

2 Division of Neonatology, Levine Children's Hospital Atrium Health, Charlotte, NC, USA

3 Health Services and Outcomes Research, Children's Mercy Kansas City; School of Medicine, University of Missouri-Kansas City, Kansas City, MO, USA

4 Biostatistics and Epidemiology Core, Health Services and Outcomes Research, Children's Mercy Kansas City; School of Medicine, University of Missouri-Kansas City, Kansas City, MO, USA for a sustained period. Neurally Adjusted Ventilator Assist (NAVA) (Getinge Group, Getinge, Sweden) is a technique of assisted neonatal ventilation that depends on diaphragm electrical signaling for initiating each respiratory effort. The technique is associated with both promise and potential challenges in this population. Previous studies have shown improvements in ventilator dynamics that may reduce ventilator-induced lung injury; however, the interaction between a structurally abnormal diaphragm and a ventilator modality dependent on diaphragmatic neural innervation is unknown and may negate any potential benefits.

NAVA uses the patient's neural respiratory drive to deliver synchronized assisted breaths, as opposed to standard ventilation modes, which use flow, pressure, or volume triggers. With NAVA, a sensor in a feeding tube at the level of the diaphragm detects the patient's respiratory rate and effort represented as the Edi Peak (electrical activity during diaphragmatic contraction) and the Edi Min (tonic resting activity between breaths). The ventilator delivers a mechanical breath to proportionally augment the patient's own respiratory effort to a degree set by the clinician as the "NAVA level". This unique approach to ventilation has been correlated with improved patient-ventilator interaction [2], 
lower peak inspiratory pressure (PIP) and reduced respiratory muscle load [3], decreased $\mathrm{FiO} 2$ and respiratory rate [4], and decreased tidal volumes [5]. Benefits have been seen with NAVA in neonates with varying disease states. However, only limited studies have explored whether these benefits extend to neonates with an abnormal diaphragm [6-8]. It is unclear whether diaphragmatic structural and neural differences interact with NAVA in a way that limits its potential benefits.

The objectives of this study are twofold. First, we sought to understand the clinical response to NAVA by neonates with CDH. NAVA was used after surgical repair, as the ventilator support was stable or being weaned. Short term outcomes such as ventilator settings, analgesic medication use, vital signs, and laboratory values, were compared preNAVA to a period of $72 \mathrm{~h}$ while on NAVA. Second, we conceptualized the effect of an abnormal diaphragm on the functioning of the NAVA ventilator by comparing NAVA Level, and both the tonic (Edi Min) and contractile (Edi Peak) electrical activity of the diaphragm between neonates with $\mathrm{CDH}$ and those without $\mathrm{CDH}$.

\section{Methods}

The neonatal intensive care unit at Children's Mercy Kansas City (CMKC) is a quaternary 84-bed unit. The local databases for the Children's Hospital Neonatal Consortium and Infant Pulmonary Data Repository, both independently approved by the CMKC Institutional Review Board, identified neonates meeting inclusion criteria and provided deidentified data for this study. NAVA has been used at our institution since 2010 in accordance with guidelines from the manufacturer, institutional experience and published clinical guidelines \& management strategies [9].

\section{Inclusion/exclusion criteria}

All infants placed on NAVA support and diagnosed with $\mathrm{CDH}$ or congenital diaphragmatic eventration between 2011 and 2019 were included. Neonates were placed on NAVA at the discretion of the attending physician. Data were analyzed only for those who were successfully maintained on NAVA for a period greater than $24 \mathrm{~h}$. Neonates with low grade or minor diaphragmatic eventrations were excluded.

\section{Protocol}

For the first objective, a retrospective cohort analysis was performed for neonates with $\mathrm{CDH}$ receiving NAVA ventilatory support. To ensure that this clinician-selected group reflected the larger population of neonates with $\mathrm{CDH}$, we used the local Children's Hospital Neonatal Consortium database to compare the neonates placed on NAVA with the larger population of neonates with $\mathrm{CDH}$ with regard to characteristics such as organ herniation, sidedness of the defect, need for extracorporeal membrane oxygenation, need for iNO, and survival to discharge. Primary outcomes for the neonates placed on NAVA included respiratory severity score $(\mathrm{RSS}=\mathrm{MAP} \times \mathrm{FiO} 2)$, PIP, MAP, PEEP, morphine-equivalent use, and midazolam-equivalent use. Secondary outcomes included vital signs and blood gases to ensure no compensatory clinical worsening while on NAVA. Values were obtained immediately before going on NAVA, and were compared to values at intervals of 1,24, and $72 \mathrm{~h}$ after NAVA in order to understand immediate and short-term changes. If data were not available at the specific interval, the closest available data within $3 \mathrm{~h}$ were used. For analgesic medication use, the cumulative daily dose was calculated $24 \mathrm{~h}$ before NAVA, $24 \mathrm{~h}$ after, and for the interval $72-96 \mathrm{~h}$ after. Analgesic medications were administered at a low hourly basal rate with the option to receive a larger PRN dose for pain or agitation. Weaning of the hourly rate was an individualized decision by the physician based on the baby's pain scores and need for PRN doses during the previous day. Opioid doses were converted to morphine-equivalents using the Lexicomp opioid conversion table. One neonate with a diaphragmatic eventration requiring surgical repair was placed on NAVA prior to surgery; the remaining neonates with $\mathrm{CDH}$ were placed on NAVA post-surgery only.

For the second objective, a case-control study was performed to describe the impact of a congenitally abnormal diaphragm on NAVA settings. A 1:2 matched control group of neonates without $\mathrm{CDH}$ was created to compare with the neonates with $\mathrm{CDH}$ identified in the first objective. Matching was performed by post-menstrual age when starting NAVA, weight when starting NAVA, and RSS $(\mathrm{MAP} \times \mathrm{FiO} 2)$ just prior to initiating NAVA. Reviewed data included NAVA Level, and the electrical activity of the diaphragm at contraction and at rest as represented by the Edi Peak and Edi Min, respectively.

\section{Data collection and statistical analysis}

For the first objective, the physiologic state of each infant was evaluated pre-NAVA and at intervals of 1,24 , and $72 \mathrm{~h}$ while on NAVA. For descriptive purposes, each patient's change from baseline on each of the ten outcome variables was computed at each subsequent time point, including median and inter-quartile ranges for these change scores. Effects of time were then formally modeled by fitting a linear mixed model for each outcome variable in SAS 9.4 (SAS Institute, Cary, NC). In each model, the outcome variable (or its natural $\log$, if needed to satisfy the model 
assumption of Normal residuals) was modeled as a function of time point, and a random intercept was included to adjust for clustering of time points for each patient. Time point was treated as a categorical variable, allowing for non-linear changes across time, and the pre-NAVA time point was set as the referent to estimate the average change from baseline at each subsequent time point.

For the second objective, continuous ventilator settings were compared using nonparametric Mann-Whitney $U$ tests. Given the rarity of some outcomes, incidence rates were analyzed using Fisher's exact tests for robustness in cases where expected counts were near zero. To validate the efficacy of the matching process, distributions of the three matching variables were compared between case and control cohorts with another two-sample Mann-Whitney $U$ test.

\section{Results}

During the study period, a total of 130 neonates were diagnosed with $\mathrm{CDH}$, in line with the expected prevalence of this disease; 104 underwent operative repair. A total of 18 neonates were placed on NAVA ventilation, of which 2 failed within $6 \mathrm{~h}$ due to clinical deterioration (tachypnea and hypercarbia) or not triggering the ventilator (Fig. 1). Of those who were successfully maintained on NAVA, the median duration was 4 days, and $79 \%$ were successfully extubated from NAVA. NAVA was used primarily while the ventilatory support was stable or being weaned, but other reasons included unfavorable ventilatory pressures on other modalities, tachypnea, or agitation. Removal from NAVA was due primarily to extubation or need for surgical procedure. The characteristics of the neonates with $\mathrm{CDH}$ and placed on NAVA were similar to those with $\mathrm{CDH}$ not placed on NAVA with regard to patch repair $(62.5 \%$ vs $57.0 \%, p=0.79)$, liver herniation $(31.3 \%$ vs. $38.1 \%, p=$ $0.78)$, left sidedness of defect ( $100 \%$ vs $82.2 \%, p=0.075)$,

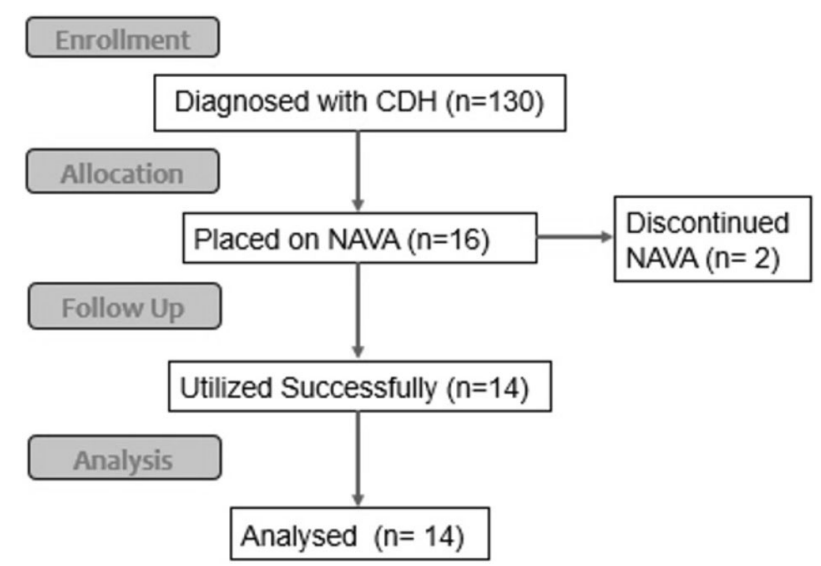

Fig. 1 CONSORT diagram showing patients included for enrollment, allocation, follow-up and analysis. need for extracorporeal membrane oxygenation (25\% vs. $27.1 \%, p=0.56)$, and survival to discharge $(87.5 \%$ vs $65.2 \%, p=0.09$ ).

For the first objective of measuring short-term outcomes of neonates with $\mathrm{CDH}$ while on NAVA, decreases were seen in ventilator indices and analgesic medication use, as compared to the period before placing on NAVA (Fig. 2). Based on mixed model estimates (Table 1), there was a decrease in PIP at $1 \mathrm{~h}[-4.4$, (95\% CI -7.6, -1.1), $p=$ $0.010], 24 \mathrm{~h}[-5.0,(-7.7,-2.4), p<0.001]$, and $72 \mathrm{~h}$ $[-8.5,(-14.0,-3.1), p=0.003] ; \operatorname{RSS}$ at $24 \mathrm{~h}[-0.6$, $(-1.1,-0.1), p=0.029]$, and $72 \mathrm{~h}[-1.4,(-2.0,-0.7)$, $p<0.001]$; MAP at $72 \mathrm{~h}[-3.0,(-4.4,-1.5), p<0.001]$; morphine-use at $72 \mathrm{~h}[-0.4 \mathrm{mg} / \mathrm{kg} / \mathrm{day},(-0.7,-0.1), p=$ $0.004]$; and midazolam use at $72 \mathrm{~h}[-0.4 \mathrm{mg} / \mathrm{kg} /$ day, $(-0.7$, $0.0), p=0.037]$. One patient was started on clonidine as the other analgesic medications were being weaned; no patients were administered methadone. There were no statistically significant changes in PEEP, heart rate, or respiratory rate, although respiratory rate transiently declined by $17 \%$ $(p=0.066)$.

For the second objective of understanding the impact of a congenitally abnormal diaphragm on NAVA settings, neonates with $\mathrm{CDH}$ and placed on NAVA were compared to a 1:2 matched control group of neonates without $\mathrm{CDH}$ who were placed on NAVA. Their clinical and demographic characteristics are compared in Table 2. There was no clinically significant difference between the two groups in NAVA level $(p=0.286)$, nor electrical activity of the diaphragm as measured by the Edi-Peak $(p=0.315)$ and EdiMin $(p=0.266)$ (Fig. 3).

\section{Discussion}

Our study demonstrates that invasive NAVA in neonates with diaphragmatic abnormalities can be used successfully in most cases when ventilatory support is stable or being weaned and is associated with improvements in ventilator dynamics such as PIP, MAP, and RSS. Our results are similar to previous literature in neonates without $\mathrm{CDH}$ that has shown NAVA to be safe and feasible [4] and associated with benefits such as improved patient-ventilator synchrony [2]. Previous small studies and case reports in neonates with CDH [6-8] have found NAVA to be associated with decreased PIPs, A-aDO2, and $\mathrm{PaCO} 2$ over $24 \mathrm{~h}$. Our study confirmed and expanded on these findings with improvements observed in PIP, MAP, and RSS over a period of $72 \mathrm{~h}$. In other more diverse populations, such as neonates with severe BPD [10] and PICU patients [11], NAVA has been associated with a decrease in sedation medication use; our study demonstrates similar findings in neonates with $\mathrm{CDH}$. 

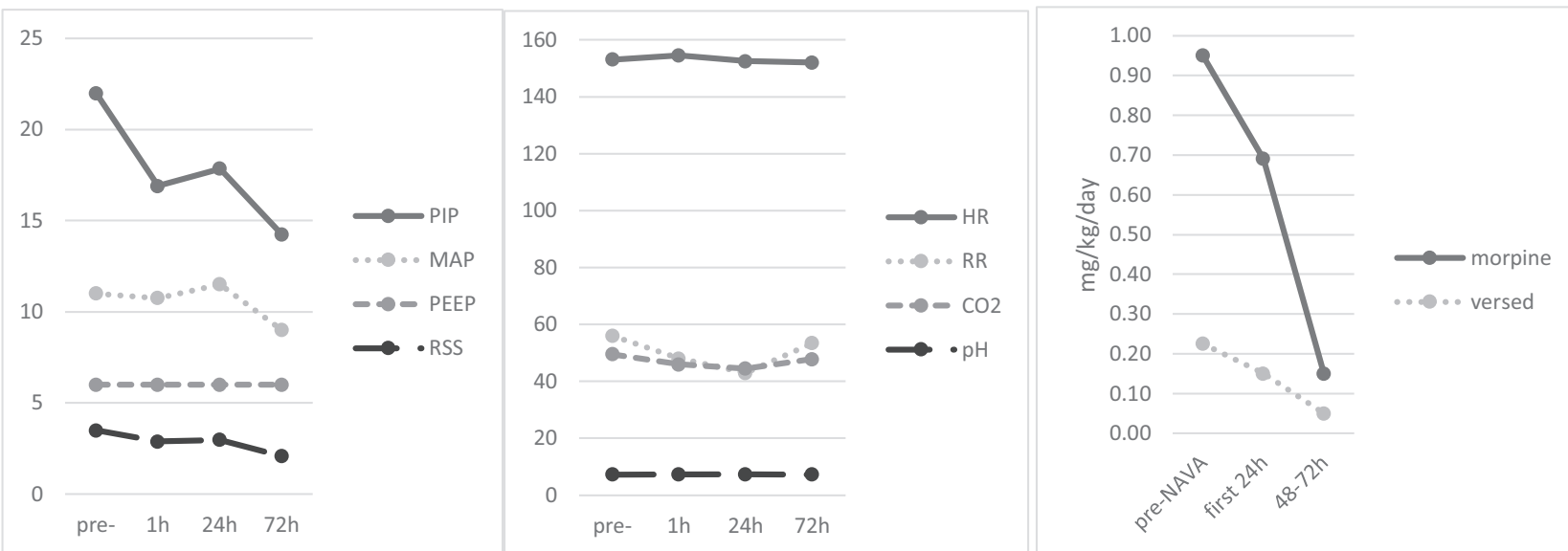

Fig. 2 Observed median at pre-NAVA at 1, 24, and 72 h. PIP Peak Inspiratory Pressure, MAP Mean Airway Pressure, RSS Respiratory Severity Score, HR Heart Rate, RR Respiratory Rate.

Table 1 Mixed model estimates of average change from pre-NAVA at 1, 24, and $72 \mathrm{~h}$.

\begin{tabular}{|c|c|c|c|c|c|c|}
\hline \multirow[b]{2}{*}{ Variable } & \multicolumn{2}{|l|}{$1 \mathrm{~h}$} & \multicolumn{2}{|l|}{$24 \mathrm{~h}$} & \multicolumn{2}{|l|}{$72 \mathrm{~h}$} \\
\hline & $\mathrm{B}(95 \% \mathrm{CI})$ & $P$ & $\mathrm{~B}(95 \% \mathrm{CI})$ & $\mathrm{P}$ & $\mathrm{B}(95 \% \mathrm{CI})$ & $P$ \\
\hline \multicolumn{7}{|l|}{ Model estimates for unstandardized variables ${ }^{\mathrm{a}}$} \\
\hline Respiratory Severity Score $(\mathrm{MAP} \times \mathrm{FiO} 2)$ & $0.1(-0.5,0.7)$ & 0.711 & $-0.6(-1.1,-0.1)$ & 0.029 & $-1.3(-2.0,-0.7)$ & $<0.001$ \\
\hline PIP & $-4.4(-7.6,-1.1)$ & 0.010 & $-5.0(-7.7,-2.4)$ & $<0.001$ & $-8.5(-13.9,-3.1)$ & 0.003 \\
\hline PEEP & 0 (NA, NA) & NA & $0.1(-0.3,0.4)$ & 0.673 & $-0.2(-0.8,0.4)$ & 0.460 \\
\hline MAP & $-1.1(-2.5,0.3)$ & 0.111 & $-0.8(-2.2,0.7)$ & 0.306 & $-3.0(-4.4,-1.5)$ & $<0.001$ \\
\hline $\mathrm{pH}$ & $-0.01(-0.04,0.02)$ & 0.636 & $0.03(-0.01,0.06)$ & 0.115 & $0.01(-0.03,0.05)$ & 0.596 \\
\hline $\mathrm{CO} 2$ & $0.0(-4.4,4.4)$ & 0.996 & $-4.0(-8.0,-0.1)$ & 0.046 & $-1.2(-6.1,3.6)$ & 0.608 \\
\hline Heart rate & $-1.0(-9.3,7.3)$ & 0.816 & $-4.6(-14.1,4.9)$ & 0.337 & $-5.4(-15.8,5.0)$ & 0.299 \\
\hline Respiratory rate (HFOV excluded) & $-6.2(-14.6,2.3)$ & 0.147 & $-9.5(-19.6,0.6)$ & 0.066 & $-5.5(-15.6,4.6)$ & 0.277 \\
\hline Morphine equivalent (mg/kg/day) & & & $-0.2(-0.4,0.1)$ & 0.268 & $-0.4(-0.7,-0.2)$ & 0.004 \\
\hline Versed equivalent (mg/kg/day) & & & $-0.1(-0.2,0.0)$ & 0.131 & $-0.3(-0.7,0.0)$ & 0.037 \\
\hline \multicolumn{7}{|l|}{ Model estimates for standardized variables ${ }^{\mathrm{b}}$} \\
\hline Respiratory Severity Score $(\mathrm{MAP} \times \mathrm{FiO} 2)$ & $0.1(-0.3,0.4)$ & 0.711 & $-0.3(-0.6,0.0)$ & 0.029 & $-0.8(-1.1,-0.4)$ & $<0.001$ \\
\hline PIP & $-0.6(-1.1,-0.2)$ & 0.010 & $-0.7(-1.1,-0.3)$ & $<0.001$ & $-1.2(-2.0,-0.4)$ & 0.003 \\
\hline PEEP & 0 (NA, NA) & NA & $0.1(-0.2,0.4)$ & 0.673 & $-0.2(-0.7,0.3)$ & 0.460 \\
\hline MAP & $-0.4(-0.8,0.1)$ & 0.111 & $-0.2(-0.7,0.2)$ & 0.306 & $-0.9(-1.4,-0.5)$ & $<0.001$ \\
\hline $\mathrm{pH}$ & $-0.1(-0.7,0.4)$ & 0.636 & $0.5(-0.1,1.1)$ & 0.115 & $0.2(-0.6,1.0)$ & 0.596 \\
\hline $\mathrm{CO} 2$ & $0.0(-0.5,0.5)$ & 0.996 & $-0.5(-1.0,0.0)$ & 0.046 & $-0.2(-0.7,0.4)$ & 0.608 \\
\hline Heart Rate & $-0.1(-0.5,0.4)$ & 0.816 & $-0.3(-0.8,0.3)$ & 0.337 & $-0.3(-0.9,0.3)$ & 0.299 \\
\hline Respiratory Rate (HFOV excluded) & $-0.4(-1.1,0.2)$ & 0.147 & $-0.7(-1.4,0.0)$ & 0.066 & $-0.4(-1.1,0.3)$ & 0.277 \\
\hline Morphine equivalent (mg/kg/day) & & & $-0.2(-0.7,0.2)$ & 0.268 & $-0.7(-1.1,-0.2)$ & 0.004 \\
\hline Versed equivalent (mg/kg/day) & & & $-0.1(-0.3,0.0)$ & 0.131 & $-0.5(-1.0,0.0)$ & 0.037 \\
\hline
\end{tabular}

${ }^{\mathrm{a}}$ Regression coefficients represent average change from baseline in original units.

${ }^{\mathrm{b}} \mathrm{Scaled}$ regression coefficient representing average change in SD units (95\% confidence interval). 
Our study is the first to conceptualize the interaction between NAVA and the congenitally abnormal diaphragm by comparing ventilator settings and indices with a 1:2 matched control group without $\mathrm{CDH}$. In our population of neonates weaning from ventilatory support, we demonstrated that the congenitally abnormal diaphragm does not require higher ventilator settings, nor do the levels exceed the normal range of settings used at our institution. We observed no meaningful difference in the electrical activity of the diaphragm as detected by the NAVA catheter.

One of the strengths of our study is that it builds on the existing literature base with a larger cohort of 16 neonates and trending their outcomes over $72 \mathrm{~h}$, a longer period of time compared to previous publications. Our study shows an association between NAVA and decreased analgesic medication use in the neonatal intensive care unit. AIn addition, our use of a control group provides a framework for understanding NAVA ventilator indices and settings in $\mathrm{CDH}$ and demonstrates no meaningful differences in diaphragmatic electrical activity, nor required ventilator settings. Due to the frequent use of NAVA at our institution, we were able to create a matched control group using three

Table 2 Patient characteristics of neonates placed on NAVA comparing those with $\mathrm{CDH}$ to those without (PMA Post Menstrual Age, RSS Respiratory Severity Score, BW Birthweight, GA Gestational Age).

\begin{tabular}{lccc}
\hline & CDH & non-CDH & $p$-value \\
\hline PMA at NAVA & $395 / 7 \pm 15$ days & $405 / 7 \pm 29$ days & 0.232 \\
Weight at NAVA & $3.36 \pm 0.775$ & $3.32 \pm 1.30$ & 0.215 \\
RSS & $4.30 \pm 3.17$ & $4.00 \pm 1.82$ & 0.380 \\
BW $(\mathrm{kg})$ & $3.09 \pm 0.631$ & $2.84 \pm 0.955$ & 0.209 \\
GA at birth & $38 / 0 \pm 9$ days & $36 / 2 \pm 28$ days & 0.064 \\
\hline
\end{tabular}

parameters (weight, PMA, and RSS) which decreases variability between the two comparison groups.

There are limitations to our study owing to sources of potential bias. First, the neonates were placed on NAVA at physician discretion and were not randomly chosen, introducing the potential for selection bias; if the selected neonates do not represent the larger population, it is possible that the neonates placed on NAVA had higher or lower disease severity compared to the larger population of neonates with $\mathrm{CDH}$. We attempted to mitigate this limitation by comparing characteristics of the study group with the larger $\mathrm{CDH}$ population and found little evidence of meaningful differences in measured characteristics. Selection bias is a potential limitation in the second objective as well, as the matching criteria may not fully account for differences between the intervention and control groups. Second, due to the retrospective nature of this study, blinding was not possible, creating the potential for observer bias. We mitigated this limitation by using objective measures such as ventilator indices, documented medication use, vital signs, and blood gases. Last, with only 16 neonates, our study is small, owing to the rarity of $\mathrm{CDH}$, to the fact that NAVA is a relatively new innovation, and to provider discretion with regard to choice of ventilator modalities.

In conclusion, our study demonstrates that the potential benefits of NAVA extend to neonates with $\mathrm{CDH}$, and we found no compensatory change in Edis, nor a need for higher/lower ventilator settings compared to neonates without $\mathrm{CDH}$. The current literature base regarding NAVA in this patient population is limited; it is difficult to make recommendations regarding patient care based on such limited evidence. Therefore, further investigation into the optimal ventilator modality for neonates with $\mathrm{CDH}$ would best be performed in a prospective, multicenter study.

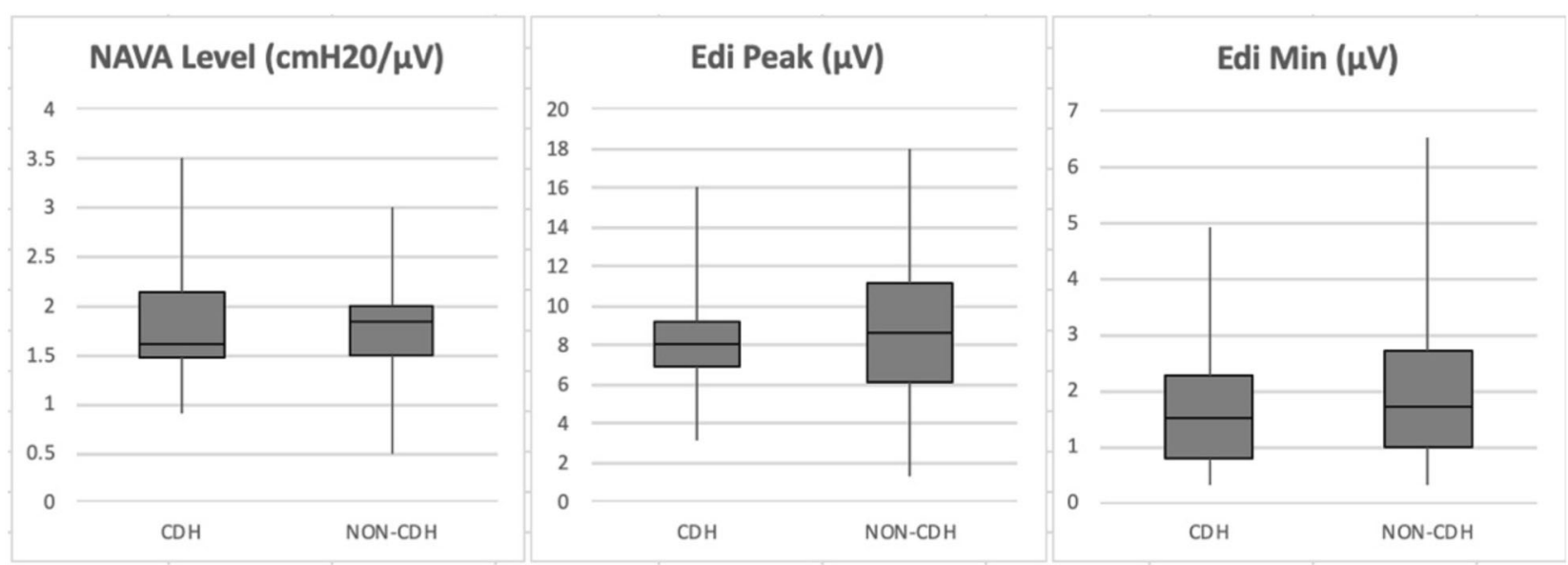

Fig. 3 Comparison of ventilator settings and respiratory indices of patients placed on NAVA with and without CDH. Edi Peak electrical activity of the diaphragm at inhalation, Edi Min tonic resting electrical activity of the diaphragm. 


\section{Data availability}

Datasets will be made available upon request.

Acknowledgements We extend our appreciation and gratitude to the following individuals and organizations: Mike Norberg and Darian Younger provided access to existing data sets acquired on behalf of the Infant Pulmonary Data Repository and Children's Hospital Neonatal Consortium (CHNC), respectively. Mike Norberg assisted with IRB approval. CHNC and Children's Mercy Research Days both provided independent opportunities to present our data as a platform presentation, and both provided clinical research awards based on the strength of the abstract and data. The Medical Writing Center at Children's Mercy Kansas City edited this manuscript.

Author contributions $\mathrm{YK}$ is the principal investigator and corresponding author. WT is the senior author. All authors provide final approval of the version for publication and agree to be accountable for all aspects of the work. YK, KG, and WT contributed to study conception. YK, KG, EP, WM, and WT designed the study and interpreted the data. YK, KF, and VS analyzed the data. YK and $\mathrm{KG}$ acquired the data and drafted the manuscript. YK, KG, EP, WM, and WT interpreted the data. All authors revised the manuscript.

\section{Compliance with ethical standards}

Conflict of interest The authors have no financial conflicts of interest.

Publisher's note Springer Nature remains neutral with regard to jurisdictional claims in published maps and institutional affiliations.

\section{References}

1. Lath NR, Galambos C, Rocha AB, Malek M, Gittes GK, Potoka DA. Defective pulmonary innervation and autonomic imbalance in congenital diaphragmatic hernia. Am J Physiol Cell Mol
Physiol [Internet]. 2012;302:L390-8. https://doi.org/10.1152/a jplung.00275.2011. Available from

2. Beck J, Reilly M, Grasselli G, Mirabella L, Slutsky AS, Dunn MS, et al. Patient-ventilator interaction during neurally adjusted ventilatory assist in low birth weight infants. Pediatr Res [Internet]. 2009;65:663-8. https://doi.org/10.1203/PDR.0b013e31819e72ab. Available from

3. Lee J, Kim H-S, Sohn JA, Lee JA, Choi CW, Kim E-K, et al. Randomized crossover study of neurally adjusted ventilatory assist in preterm infants. J Pediatr [Internet]. 2012;161:808-13.e2. https://doi.org/10.1016/j.jpeds.2012.04.040. Available from

4. Stein H, Alosh H, Ethington P, White DB. Prospective crossover comparison between NAVA and pressure control ventilation in premature neonates less than 1500 grams. J Perinatol [Internet]. 2013;33:452-6. https://doi.org/10.1038/jp.2012.136. Available from

5. Longhini F, Ferrero F, De Luca D, Cosi G, Alemani M, Colombo $\mathrm{D}$, et al. Neurally adjusted ventilatory assist in preterm neonates with acute respiratory failure. Neonatology. 2015;107:60-7.

6. Durrani NURR, Chedid F, Rahmani A. Neurally adjusted ventilatory assist mode used in congenital diaphragmatic hernia. J Coll Physicians Surg Pak. 2011;21:637-9.

7. Gentili A, Masciopinto F, Mondardini MC, Ansaloni S, Reggiani MLB, Baroncini S. Neurally adjusted ventilatory assist in weaning of neonates affected by congenital diaphragmatic hernia. J Matern Neonatal Med. 2013;26:598-602.

8. Oda A, Lehtonen L, Soukka H. Neurally adjusted ventilatory assist can be used to wean infants with congenital diaphragmatic hernias off respiratory support. Acta Paediatr Int $\mathbf{J}$ Paediatr. 2018;107:718-9.

9. Stein H, Firestone K. NAVA ventilation in neonates: Clinical guidelines and management strategies. Neonatol Today [Internet]. 2012;7:1-10. https://www.neonatologytoday.net/newsletters/nt-a pr12.pdf

10. Lee J, Kim HS, Jung YH, Choi CW, Jun YH. Neurally adjusted ventilatory assist for infants under prolonged ventilation. Pediatr Int. 2017;59:540-4.

11. Kallio M, Peltoniemi O, Anttila E, Pokka T, Kontiokari T. Neurally adjusted ventilatory assist (NAVA) in pediatric intensive care — a randomized controlled trial. Pediatr Pulmonol. 2015;50:55-62. 